\title{
Laparoscopic Splenectomy: case series of 24 patients
}

Vikal Chandra Shakya, Bishesh Paudyal

Department of Surgery, Civil Service Hospital, Minbhawan, Kathmandu

Correspondence : Vikal Chandra Shakya; Specialist, Department of Surgery, Civil Service Hospital, Minbhawan, Kathmandu.

Email: vikalcsh@yahoo.com

\section{ABSTRACT}

Introduction and Objective: Splenectomy has mostly been performed by open surgery. Laparoscopy seems to be a meaningful alternative to open technique.

Materials and Methods: This study was done in patients presenting to hematology and surgery department of Civil Service Hospital who underwent laparoscopic splenectomy from January 2013 to November 2015

Results: There were 24 patients ( 16 females, 8 males). The diagnoses were idiopathic thrombocytopenic purpura in 19, hereditary spherocytosis in 2, @hemolytic anemia in 2, b-hemolytic anemia in 1 . The mean operative time was $130+49$ minutes. The mean postoperative stay was $5+2.11$ days. Laparoscopic splenectomy could be completed in 21 patients. Rest 3 needed conversion to open (causes being excessive bleeding form splenic vein, splenic tear, and excessively low platelet counts). Three patients needed additional pfanneinsteil/lumbar incision to retrieve the spleen.

Conclusion: Laparoscopic splenectomy could be successfully contemplated in patients with hematological diseases, more so if spleen is of normal or mildly enlarged; and could be an advantageous alternative to open splenectomy.

Key words: laparoscopic splenectomy; thrombocytopenic purpura, hemolytic anemia 